\title{
Fundus autofluorescence and multicolour imaging comparison in assessing disease severity in patients suffering from geographic atrophy. A case series analysis
}

\author{
Ioannis Mallias, Panagiota Mylova, Anastasia Tassiopoulou \\ Laser Plus Eye, Nea Smyrni, Athens, Greece
}

\begin{abstract}
INTRODUCTION. The aim of the study is to determine which depicting modality, fundus autofluorescence or multicolour imaging, is a better diagnostic tool in the monitoring of the progression of geographic atrophy in patients with severe dry macular degeneration.

MATERIAL AND METHODS. Fifty patients (93 eyes) participated in this study. OCT examination (Spectralis HRA + OCT, Heidelberg Engineering, Germany) was performed in order to confirm that they fit the criteria to proceed in the study. In all patients, fundus autofluorescence and multicolour imaging was performed.

RESULTS. The area of geographic atrophy was depicted with both modalities and useful information was obtained. In some cases, multicolour imaging provided more useful clues and in other cases the fundus autofluorescence provided useful information for the progression of the disease.

CONCLUSIONS. Both modalities provide a wide range of useful information about the progression of geographic atrophy in patients with severe dry age-related macular degeneration and should both be performed in order to gather all the useful information about the disease progression.
\end{abstract}

KEY WORDS: dry age-related macular degeneration, geographic atrophy, multicolour, fundus autofluorescence

Ophthalmol J 2017; Vol. 2, No. 3, 69-77

\section{INTRODUCTION}

Age-related macular degeneration (AMD) is an acquired degeneration of the retina that causes significant central visual impairment $[1,2]$. AMD is the most common cause of irreversible vision loss in the developed world. The disease manifests with two types: the non-neovascular or dry type, which includes the formation of drusen and retinal pigment epithelium abnormalities, and or wet type, which includes a choroidal neovascular membrane formation [1-3]. Approximately $85 \%$ to $90 \%$ of the cases of macular degeneration are dry type. Dry, age-related macular degeneration does not involve any leakage of blood or serum.
Drusen are small white or yellowish deposits that form under the retina $[4,5]$, beneath the macula, causing it to deteriorate or degenerate over time. These small yellow deposits $[6,7]$ beneath the retina are a build-up of metabolic waste materials. They are made up of cholesterol, protein, and fats. Typically, when they first form, they do not cause any vision loss. However, they are a risk factor for progression to vision loss. These small yellowish deposits become larger as the disease progresses. As dry AMD progresses [8] to retinal atrophy and central retinal degeneration, loss of central vision is often observed. Nearly all people over the age of 50 years have at least one small drusen in one or both eyes [9]. 
Generally, dry AMD has a much slower, progressive visual loss relative to wet AMD.

No one knows exactly what causes macular degeneration. However, several studies [10] indicate it may be related to a combination of hereditary and environmental factors, including smoking and diet. There are also studies indicating that people of European ancestry and lighter eye colour may face a greater risk for AMD.

Dry macular degeneration symptoms usually develop gradually [11]. They may include: visual distortions, such as straight lines seeming bent, reduced central vision in one or both eyes, increased blurriness of printed words, the need for brighter light when reading or doing close work, increased difficulty adapting to low light levels, decreased intensity or brightness of colours, and difficulty recognising faces.

Funduscopically [11], in early to intermediate dry AMD there is significant presence of multiple drusen. In intermediate dry AMD, drusen may appear confluent with significant pigment changes and pigment accumulation in the posterior pole. In addition, the retinal pigment epithelium (RPE) often appears atrophic, with easier visualisation of the underlying choroid vascular plexus. In advanced stages of dry AMD, there is coalescence of focal islands of atrophy and formation of large zones of atrophy that severely affect vision. Geographic atrophy (GA) is a chronic progressive degeneration of the macula as part of the late stage of dry AMD. The disease is characterised by localised atrophy of retinal tissue and choriocapillaris leading to central scotomas and permanent loss of visual acuity.

In patients diagnosed with AMD, an Amsler grid [12] should be used, in order to check on a weekly basis if they observe any changes on it, so as to seek professional opinion if they do. The progress of the disease should also be closely monitored by an ophthalmologist every 3-6 months. Optical coherence tomography (OCT) [13] can be used in order to observe the progress of the disease. Also, a fluorescein angiography may be performed when the disease has recently been diagnosed or the patient experiences sudden vision deterioration, in order to check if a vessel leakage has occurred and therefore the disease has progressed into wet AMD type.

Studies suggest that eating antioxidant-rich foods [14], such as fresh fruits and dark green leafy vegetables (such as kale, collard greens, and spinach), may delay the onset or reduce the severity of dry AMD. These types of vegetables contain lutein, which may protect the macula from sun damage, just as it protects the leaves of the vegetables from sun damage. Also, eating at least one serving of fatty fish per week may also delay the onset or reduce the severity of dry AMD. These types of fish are high in omega-3 fatty acids, which help decrease inflammation and promote eye health. Regular exercise also has many known health benefits. By exercising frequently, the danger of obesity, which can be associated with the appearance of AMD, diminishes. Using absorbent sunglasses is also a detrimental precaution to maintain eye health.

Fundus autofluorescence (FAF) [15-17] is a noninvasive imaging technology that gives information on the distribution of lipofuscin within the retinal pigment epithelium. Accumulation of lipofuscin within retinal pigment epithelial cells is known to be involved in the pathogenesis of age-related macular degeneration. Fundus autofluorescence imaging is a useful technique to identify high-risk characteristics in patients with dry AMD. It also gives some valuable knowledge and clues in differential diagnosis of wet AMD.

\section{MATERIAL AND METHODS}

Fifty patients (93 eyes) were examined with a Spectralis HRA + OCT (Heidelberg Engineering, Germany). OCT examination was performed in order to confirm that the participants were suffering from GA. In OCT imaging, there was not any hyper-reflective band that could be attributed to scarring from previous choroidal neovascularisation in any patient. All the participants were thoroughly examined with a slit lamp and funduscopically by Dr Mallias. Best corrected visual acuity (BCVA) was measured and multicolour and fundus autofluorescence imaging with the Spectralis HRA + OCT were obtained, in order to compare which imaging setting offers the most valuable information and assesses progression of the disease for patients who suffer from GA. The mean age of the participants was 80 years, and their mean BCVA was $20 / 40$ in the Snellen visual acuity chart.

\section{RESULTS}

The comparison between the two methods was made empirically. Dr Mallias and his team assessed all the cases of the patients enrolled, trying to evaluate which module provides more useful information in everyday practice. Below we present several cases of patients with GA in at least one eye, and 
we compare multicolour imaging and fundus autofluorescence imaging in their ability to estimate the extent of the retinal damage from GA. These cases were chosen as the most interesting ones, regarding the comparison between the two modalities.

\section{PATIENT 1}

Female, 91 years old, diagnosed with dry AMD on both eyes. Her BCVA was 20/50 on both eyes (Fig. 1 and 2).

This patient of GA had foveal spearing. Multicolour imaging shows a central area of the ret-

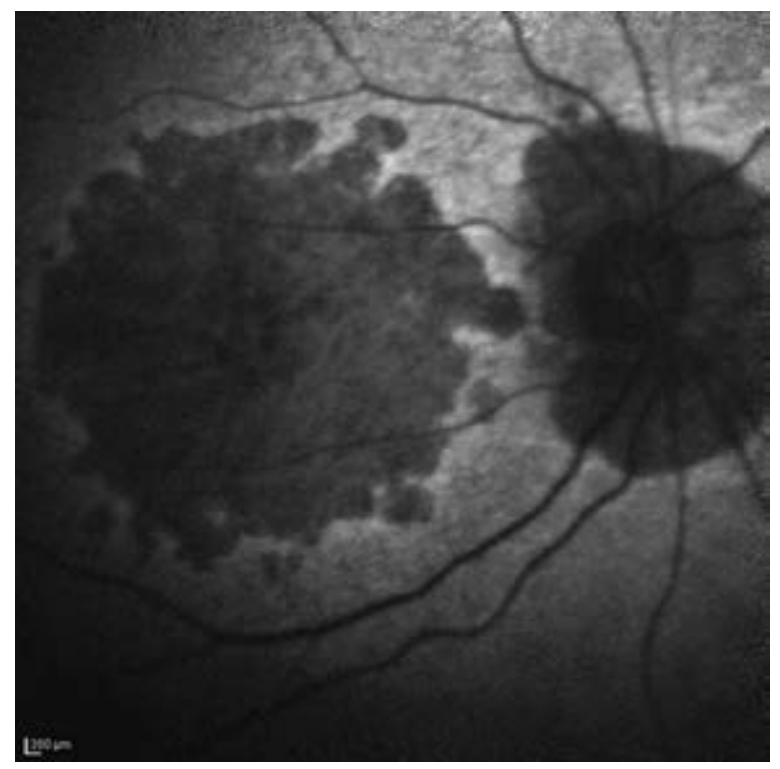

FIGURE 1. The right eye of the first patient
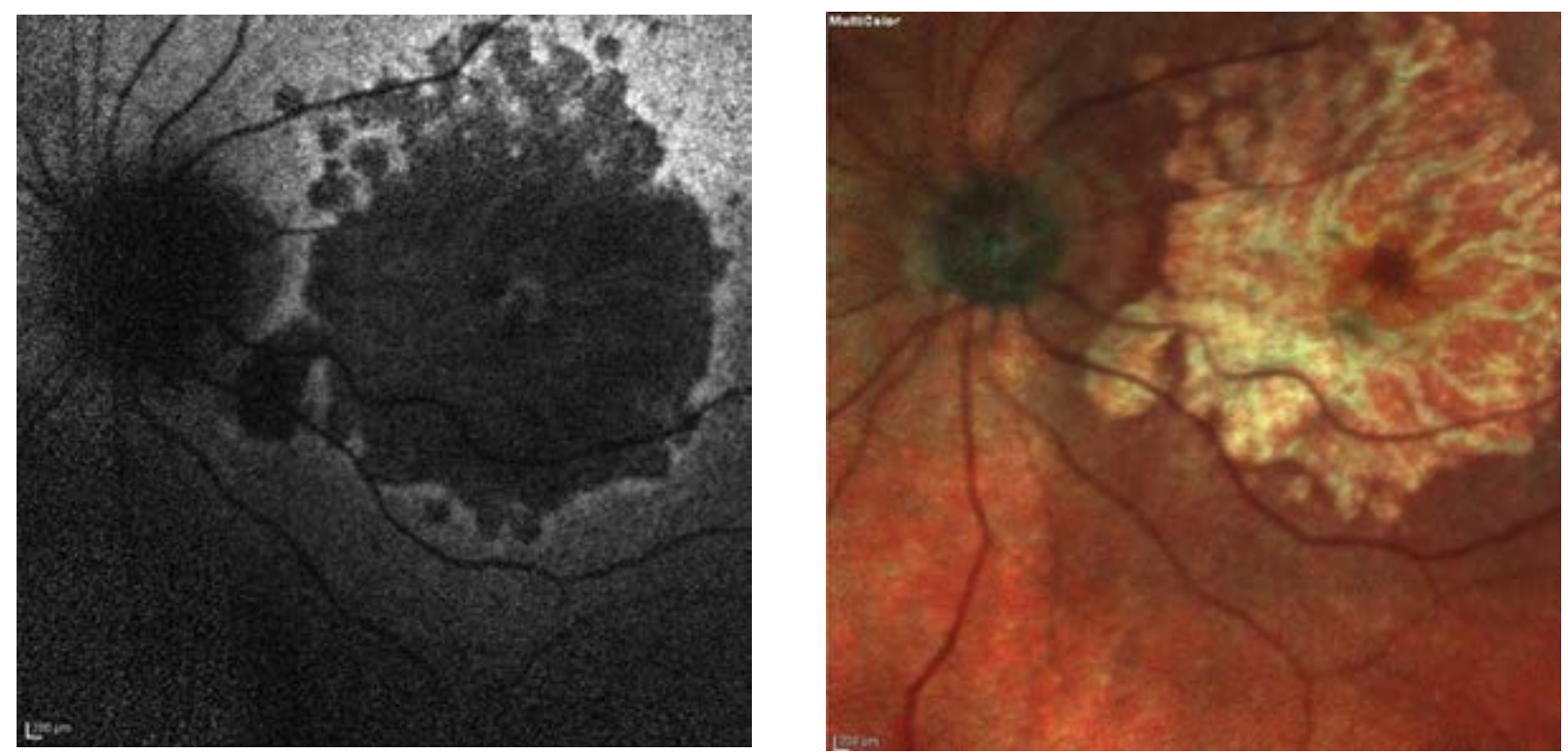

ina, close to the fovea, with no atrophy despite the extensive retinal atrophy surrounding this area. The foveal sparing could not be depicted by the autofluorescence imaging. For this particular patient, multicolour imaging was superior to fundus autofluorescence in imaging the central retinal area, which was not affected by GA.

\section{PATIENT 2}

Male, 80 years old, with dry AMD on both eyes. His BCVA was 20/25 on the right eye and 20/32 on the left eye (Fig. 3 and 4).

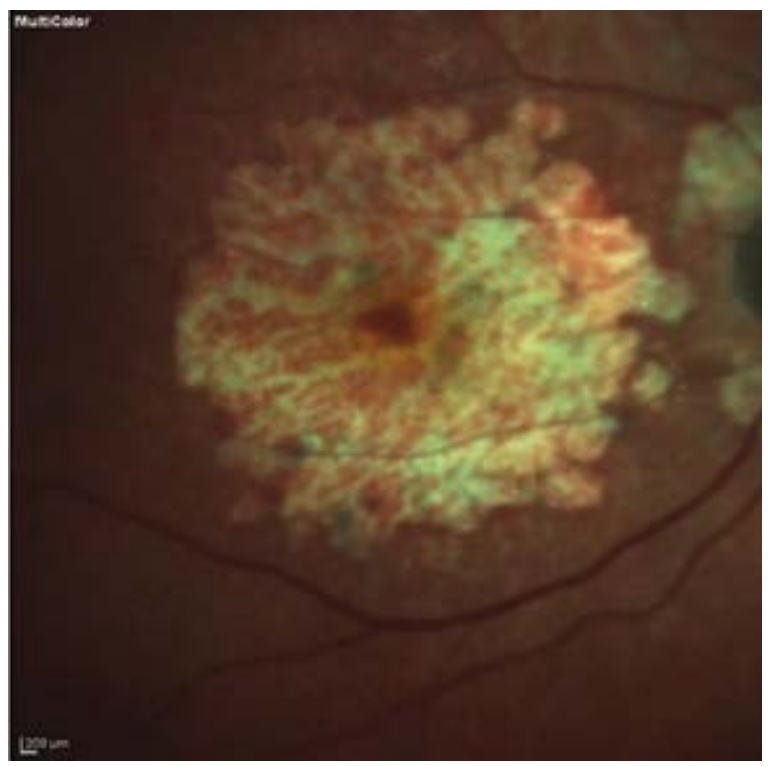

FIGURE 2. The left eye of the first patient

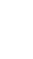



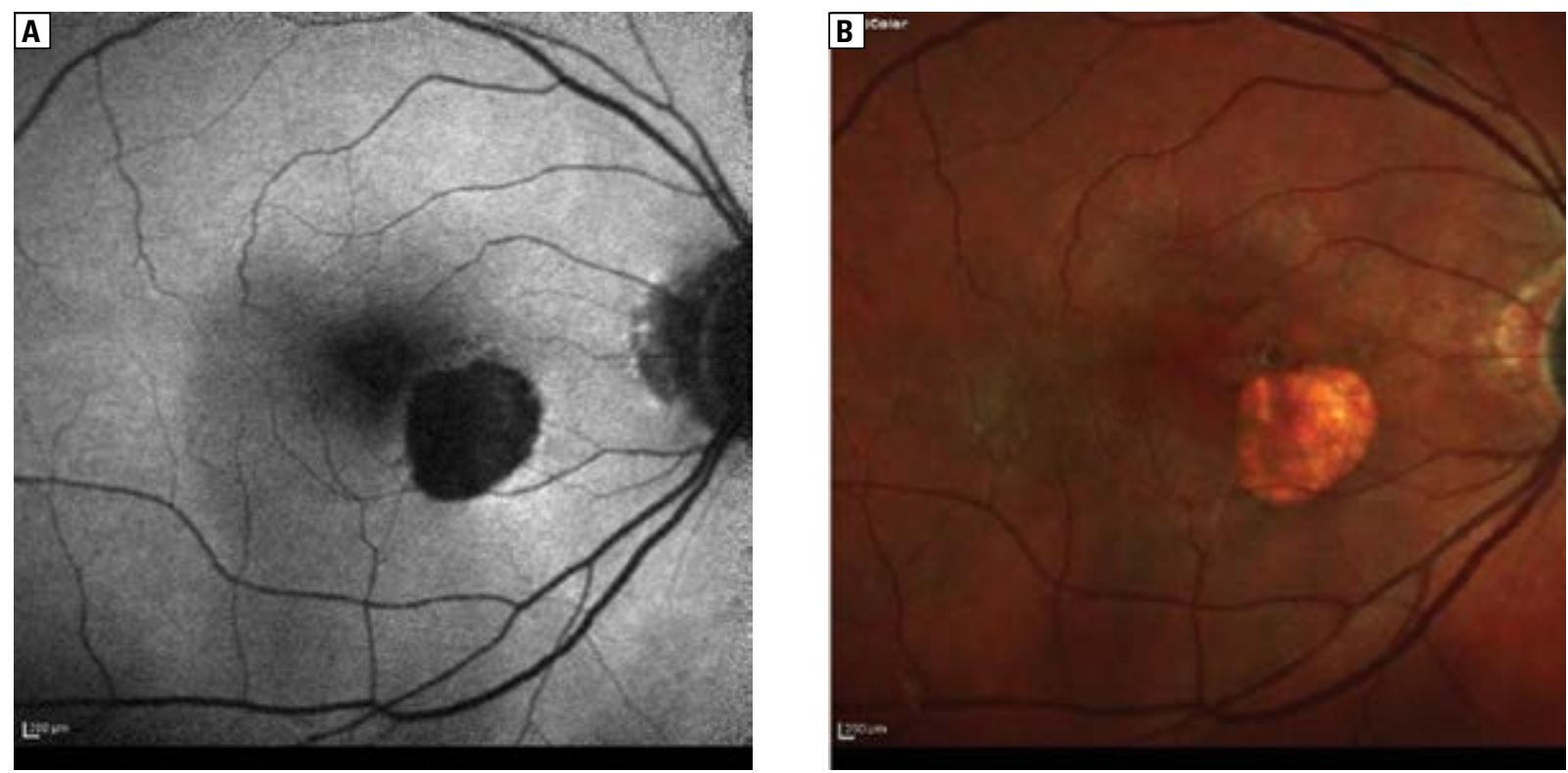

FIGURE 3. Images of the right eye of the second patient, captured with autofluorescence (A) and multicolour module (B). In both imaging modalities, a paracentral area of retinal atrophy can be seen. The extent of the retinal atrophy appears to be the same in both imaging modalities. From the fundus autofluorescence image, the fact that the retinal atrophy has not yet reached the foveal vascular zone is better depicted

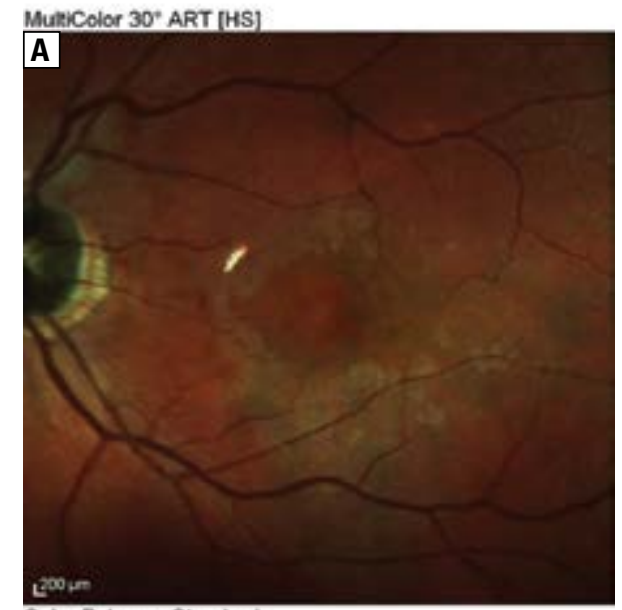

Color Balance; Standard

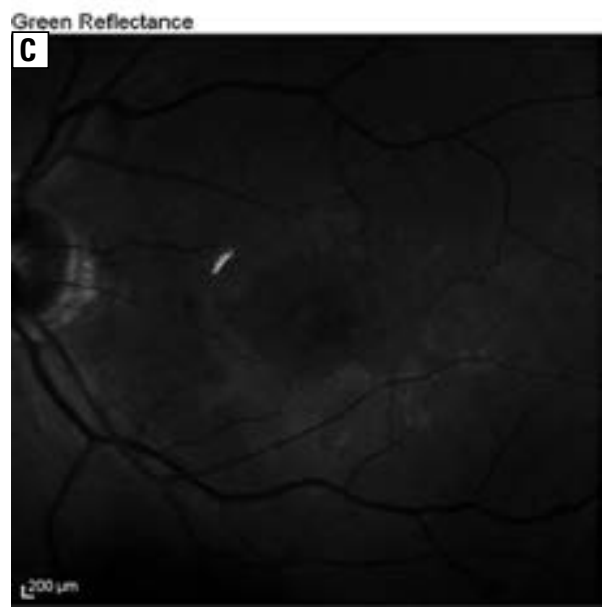

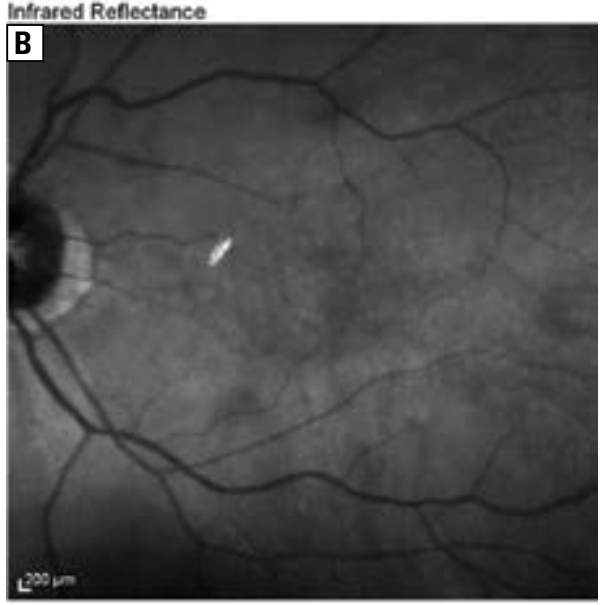

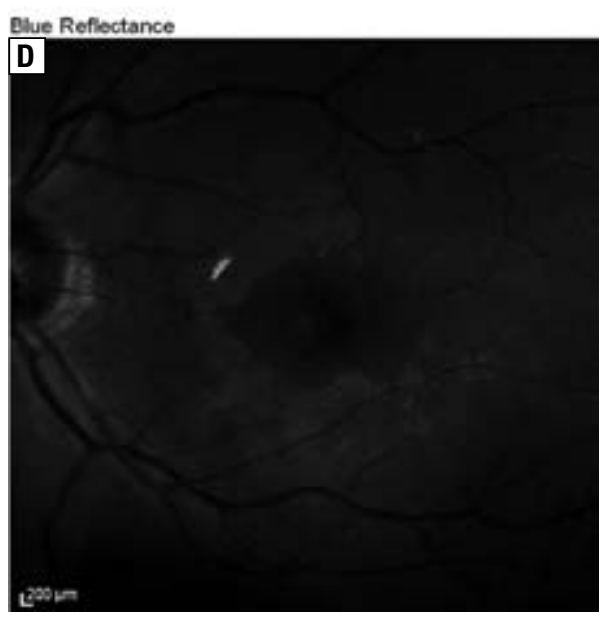

FIGURE 4. The left eye of the second patient. Multicolour imaging (A) shows a circular paracentral area with greyish colour, which reflects an area of retinal pigment epithelium abnormalities. This area in depicted in both green and blue reflectance but not in infrared (B). Also, this area is not depicted with fundus autofluorescence (C and $\mathbf{D}$ ) 


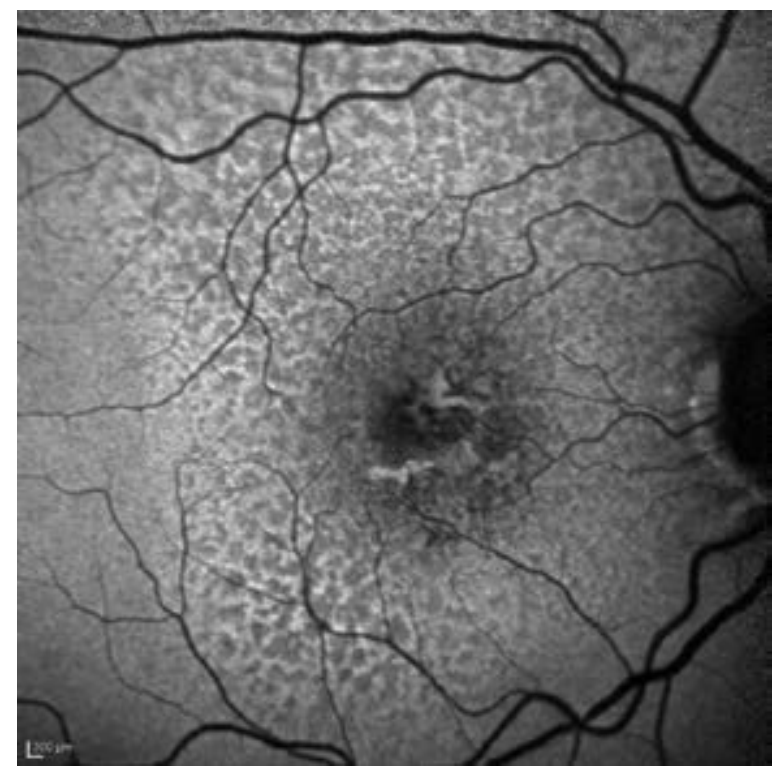

FIGURE 5. The right eye of the third patient

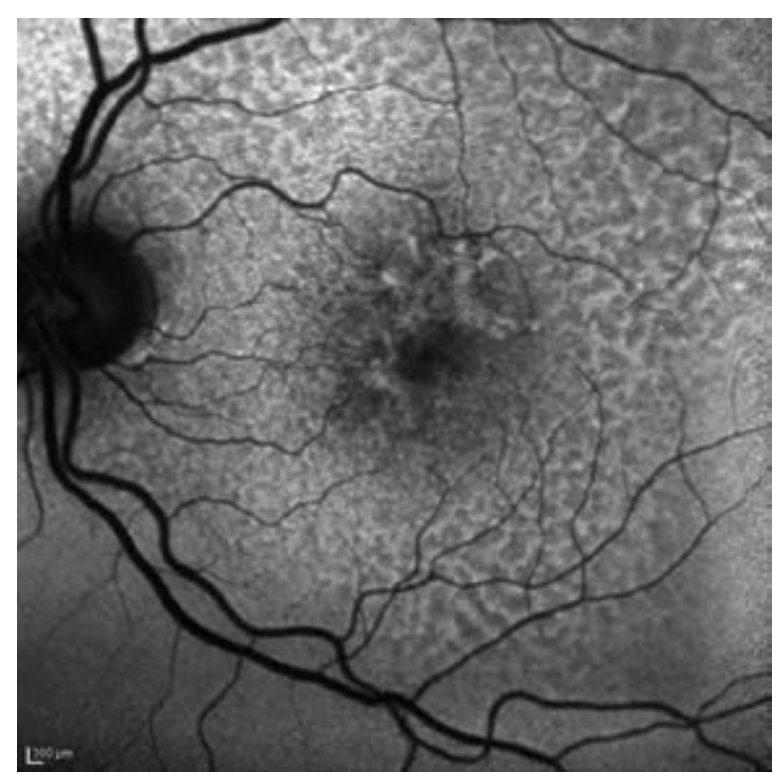

FIGURE 6. The left eye of the third patient

\section{PATIENT 3}

Female, 82 years old, diagnosed with dry AMD on both eyes. Her BCVA was 20/30 on both eyes (Figs. 5 and 6).

This patient did not have GA, but had dry AMD with reticular pseudodrusen in the parafoveal area and hard drusen at the foveal centre. Reticular pseudodrusen are better depicted with fundus autofluorescence. Hard drusen at the foveal centre are depicted with dark orange colour in multicolour imaging.
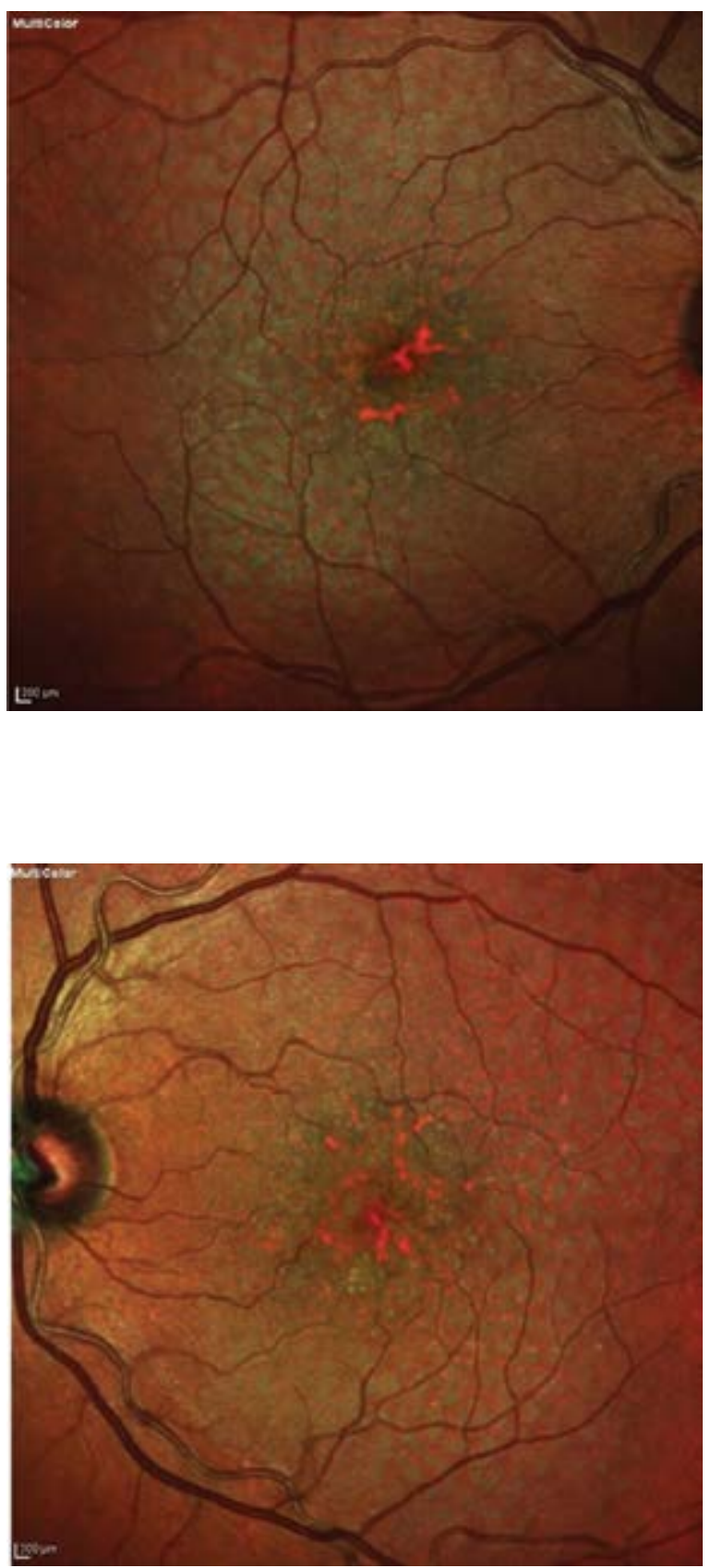

\section{PATIENT 4}

Female, 86 years old, with dry AMD on both eyes. Her BCVA was 20/30 on her right eye and 20/200 on her left (Fig. 7 and 8).

\section{PATIENT 5}

Female, 83 years old, diagnosed with dry AMD on both of her eyes. Her BCVA was 20/100 on the right eye and 20/40 on the left eye (Fig. 9 and 10).

In this patient, both imaging modalities showed the paracentral area of retinal atrophy from GA. 

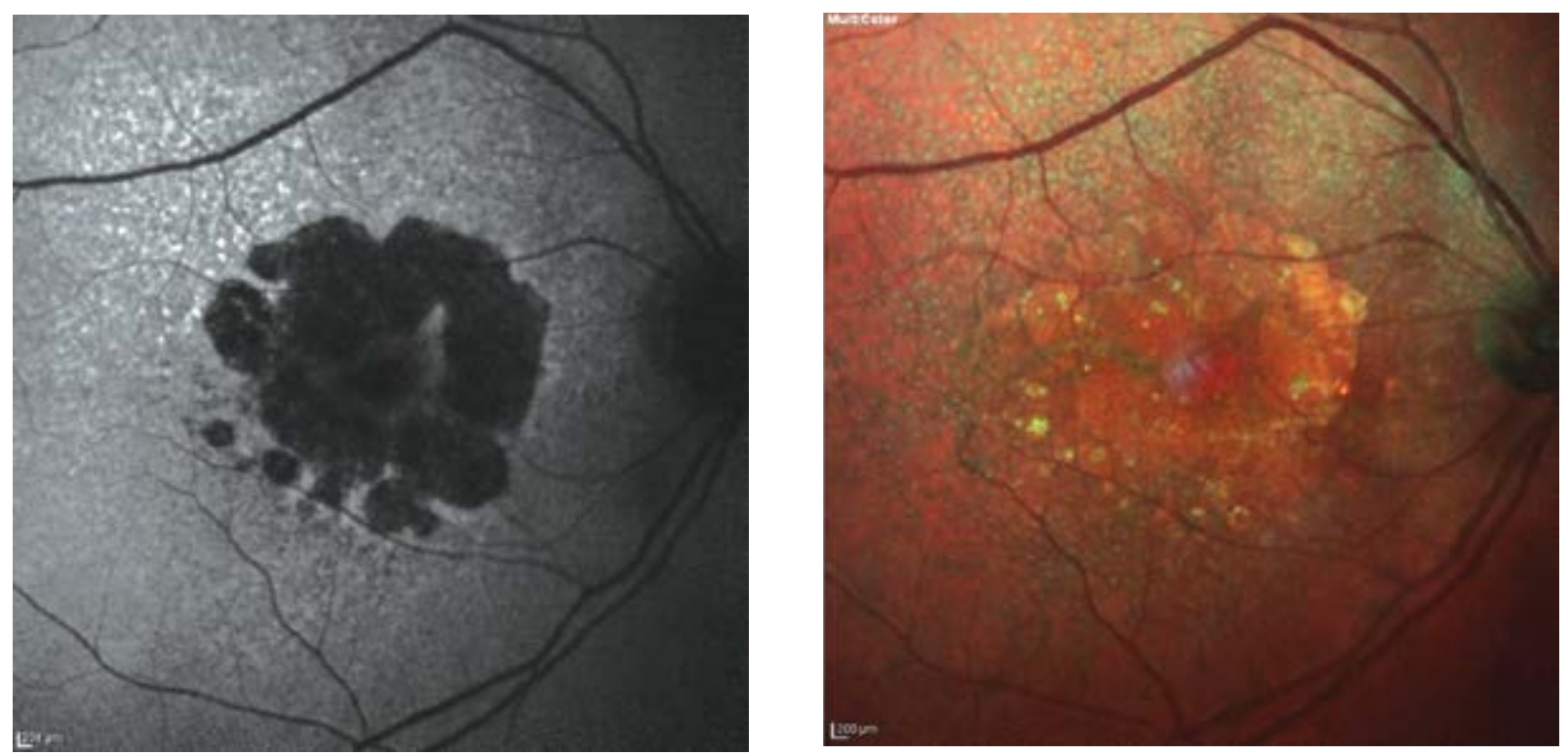

FIGURE 7. The right eye of the fourth patient. There is an extensive area of retinal atrophy with foveal sparing, and this is why the visual acuity remains quite good. Again, foveal sparing was better depicted with multicolour imaging than fundus autofluorescence
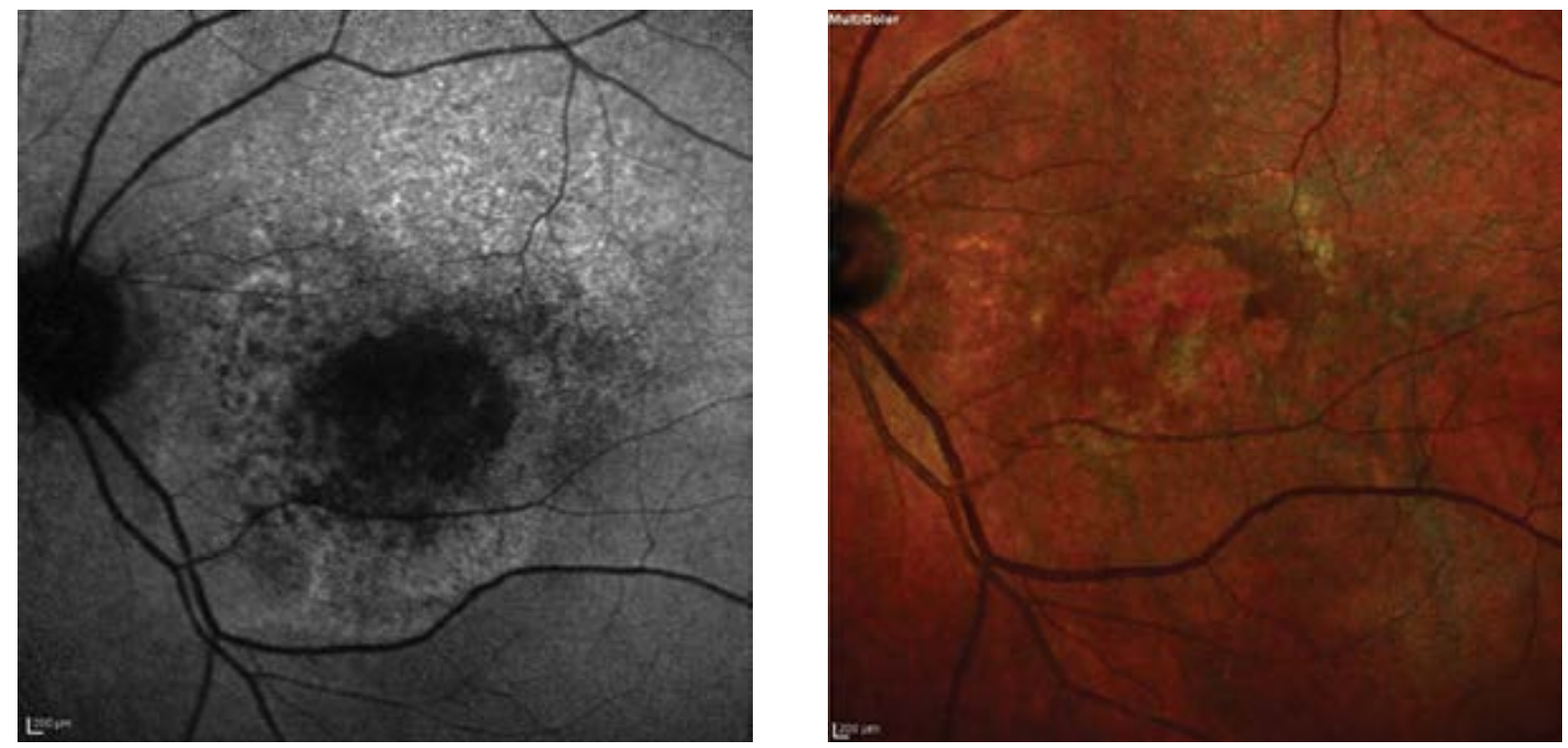

FIGURE 8. The left eye of the fourth patient. Although the area of retinal atrophy was smaller compared to the right eye, visual acuity was worse because foveal photoreceptors were already damaged by the disease. The lesions peripherally to the area of retinal atrophy were better depicted with fundus autofluorescence compared to multicolour imaging

The visual acuity of the right eye was much worse compared to the visual acuity of the left eye. With fundus Autofluorescence observed that there were already lesions from the disease, affecting the foveal avascular zone (FAZ), compared to the left eye where no lesions of the FAZ were observed with fundus autofluorescence. Despite the fact that this was a case of foveal sparing, FAF was superior to multicolour imaging for this particular case.

\section{DISCUSSION}

It is known that, unfortunately, there is no current treatment for GA. However, there was a study [18] (Spectri) from Roche (Switzerland) on the use of a substance called lampalizumab, which was investigated to determine its effect on the progression of geographic atrophy associated with advanced dry AMD. Unfortunately, Roche officially stopped the study due to poor results compared to the "placebo" 


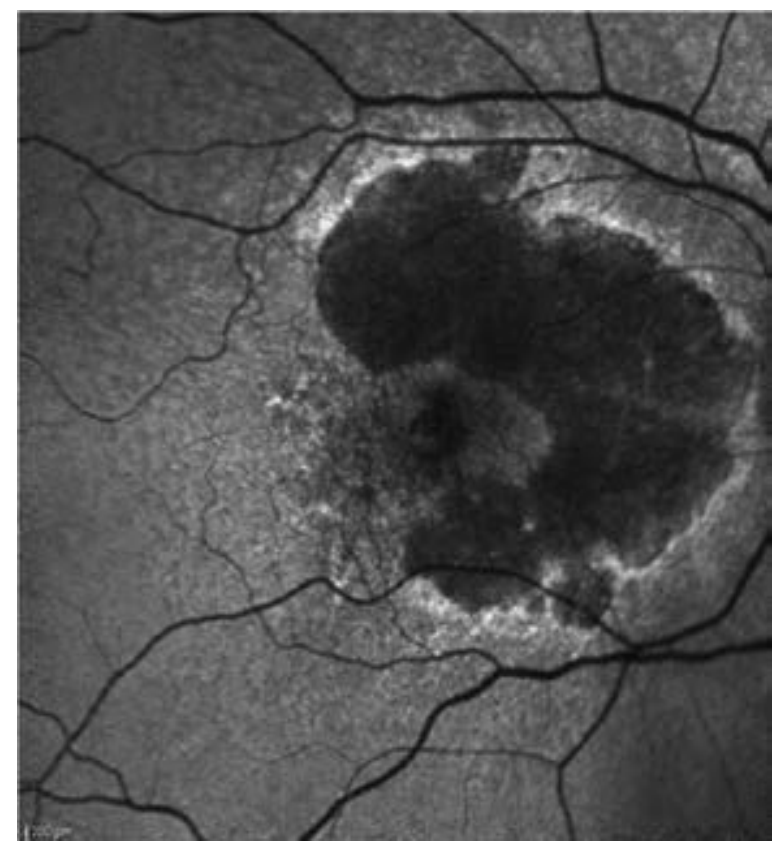

FIGURE 9. The right eye of the fifth patient

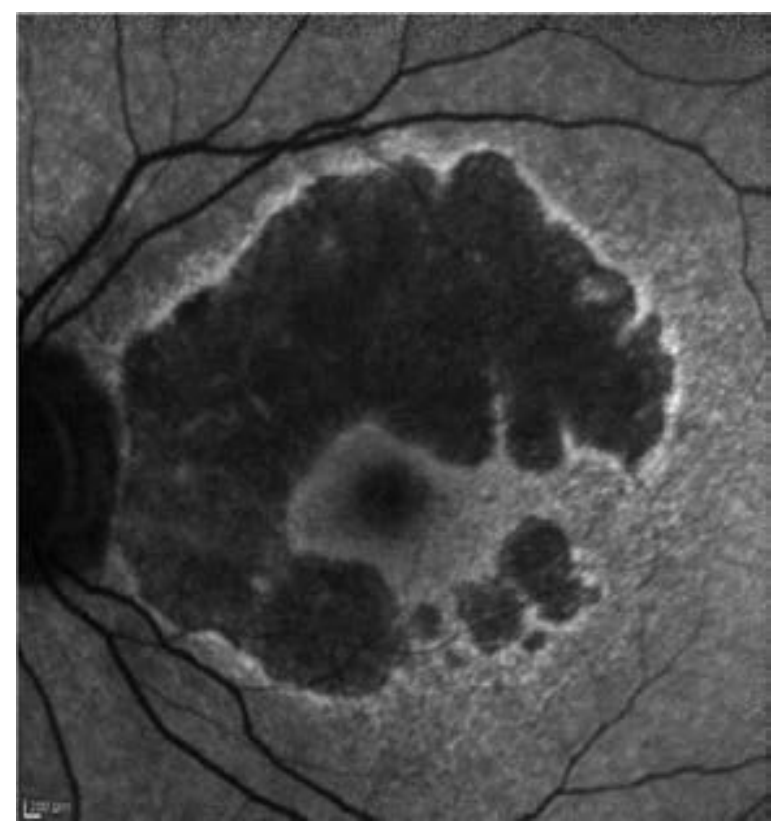

FIGURE 10. The left eye of the fifth patient

group. The company now is waiting for the results of an identically designed study (Chroma), which will be announced in November 2017.

Another solution that can give some function back to patients with geographic atrophy are: Scharioth Macula Lens [19, 20] and Implantable Miniature Telescope $[21,22]$ for macular degeneration. A Scharioth Macula Lens is an intraocular lens placed on the sulcus and it has a central addition
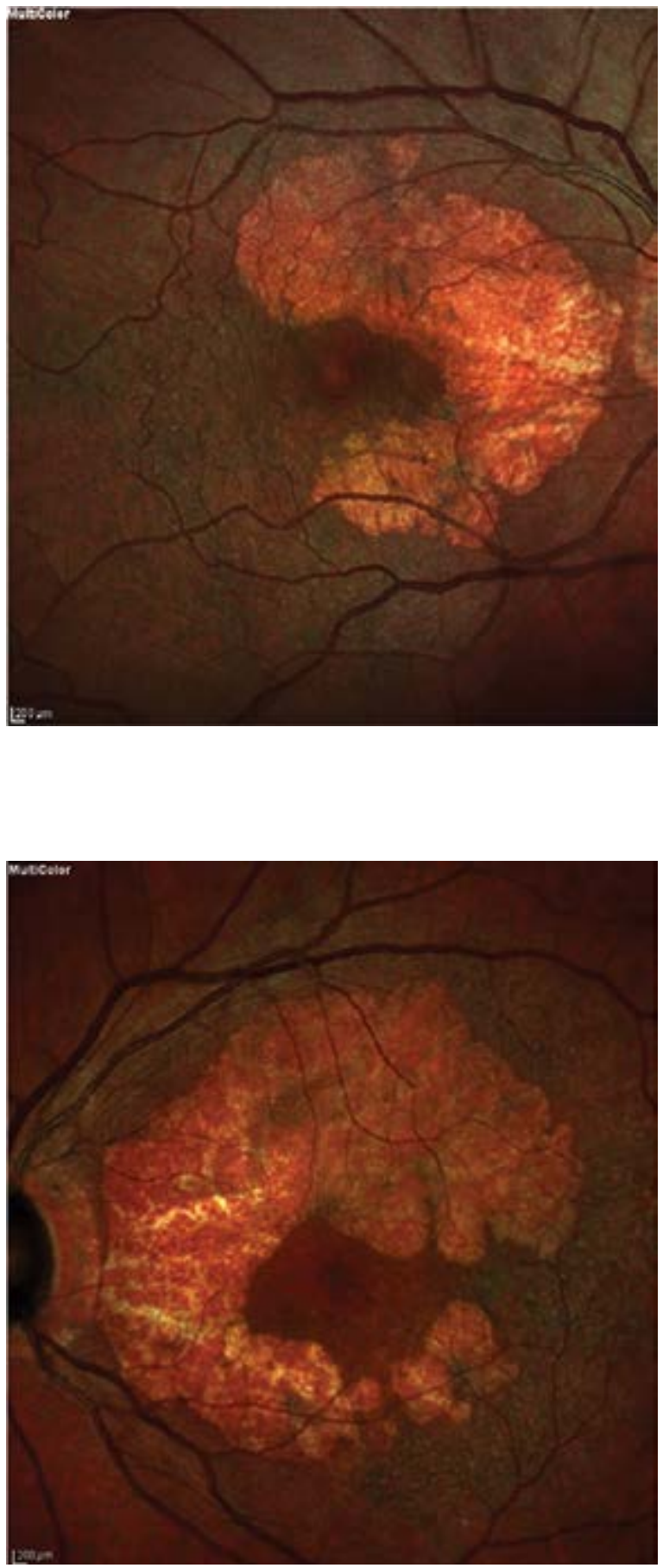

of +10 dioptres, on a diameter of $12 \mathrm{~mm}$. This lens helps patients with severe dry AMD to read through the central addition because it offers a magnification two times the size of the original image. The lens is placed on the dominant eye, or in cases where the visual acuity is very poor, the lens is placed on the eye with the best visual acuity. In order to proceed with the implantation of the Scharioth Macula Lens, the patient should already be operated for 
cataract in the imminent eye, and the intraocular lens should be placed on the capsular bag. In order for a patient to be happy with the outcome of the Scharioth Macula Lens, he/she should understand and accept that the reading distance that the lens can offer is $15 \mathrm{~cm}$. The Scharioth Macula Lens does not affect distant vision.

The implantable miniature telescope (IMT) takes advantage of the fact that even in severe dry AMD, when geographic atrophy and scarring occurs, the peripheral retina is usually not affected. Thereby, it enlarges objects in the centre of the visual field so they can be seen by the intact parts of the retina around the macula. The IMT involves patients with corrected vision of 20/160-20/800. An IMT is implanted instead of the standard plastic intraocular lens, so the patient should not have undergone cataract surgery beforehand. There are two models of IMT available: one with 2.2-times magnification and the other with 2.7-times magnification. This higher magnification version provides better visual acuity but slightly smaller visual field (20 vs. 24 degrees). IMT causes a decrease in depth perception, but this can improve with learning and experience over time.

From the images of the patients presented, it is obvious that multicolour imaging provides a better image of foveal sparing in GA. However, there were cases (such as patient 5) where the lesions progressed closer to the fovea. In these cases, FAF modality provides a better image and gives an explanation for the diminished visual acuity. Concluding, as far as multicolour imaging and FAF are concerned in the monitoring of GA, there cannot be a clear selection. Both modalities provide useful information and should both be performed in order to obtain as much information as possible.

The comparison of the two methods in all the patients was made empirically, as has already been reported. The reason for this is that in everyday practice it is not easy to take the time to actually measure and evaluate all the information that especially FAF has to offer (such as the depth and width of the atrophy). The goal of this paper was to show that it is possible to evaluate the severity of the GA of a patient when he/she first arrives at the practice by performing these two easy and fast examinations. Of course, if it is a returning patient who has been examined in the practice previously, the depth and width of the atrophy should be measured in order to accurately evaluate the disease progression.
There are several studies that compare FAF to fundus cameras [23-26], confocal scanning laser ophthalmoscope [23-25] (cSLO), and spectral-domain optical coherence tomography [27, 28] (sd-OCT) as well as studies comparing multicolour imaging to fundus photography [29], but to our knowledge there has been no study comparing FAF to multicolour imaging.

As medicine and technology develop rapidly, it would not be surprising if in the following years, one of the substances that is being tested by numerous companies has an active effect on GA. Until then, technology offers ophthalmologists and low-vision aid practitioners many tools in order to improve the quality of life of patients with GA.

\section{REFERENCES}

1. Coleman HR, Chan CC, Ferris FL, et al. Age-related macular degeneration. Lancet. 2008; 372(9652): 1835-1845, doi: 10.1016/S01406736(08)61759-6, indexed in Pubmed: 19027484.

2. Girmens JF, Sahel JA, Marazova K. Dry age-related macular degeneration: A currently unmet clinical need. Intractable Rare Dis Res. 2012; 1(3): 103-114, doi: 10.5582/irdr.2012.v1.3.103, indexed in Pubmed: 25343081.

3. Gregory S. Hageman, Karen Gehrs, Lincoln V. Johnson, and Don Anderson, Age-Related Macular Degeneration (AMD), Webvision: The Organization of the Retina and Visual System [Internet].

4. Jager RD, Mieler WF, Miller JW. Age-related macular degeneration. N Engl J Med. 2008; 358(24): 2606-2617, doi: 10.1056/NEJMra0801537, indexed in Pubmed: 18550876.

5. Rickman CB, Farsiu S, Toth C, et al. Dry age-related macular degeneration: Mechanisms, therapeutic targets, and imaging. Investigative Opthalmology \& Visual Science. 2013; 54(14): ORSF68, doi: 10.1167/ iovs.13-12757.

6. Taskintuna I, Elsayed ME, Schatz P. Update on cinical trials in dry age-related macular degeneration. Middle East Afr J Ophthalmol. 2016; 23(1): 13-26, doi: 10.4103/0974-9233.173134, indexed in Pubmed: 26957835.

7. Zhang GuM, Chen Y. Pathogenesis of dry age-related macular degeneration: updates. Zhonghua Yan Ke Za Zhi. 2014; 50(6): 464-470, indexed in Pubmed: 25241979.

8. Nowak JZ. AMD - the retinal disease with an unprecised etiopathogenesis: in search of effective therapeutics. Acta Pol Pharm. 2014; 71(6): 900-916, indexed in Pubmed: 25745762.

9. Klein R, Klein B, Moss S, et al. The Beaver Dam Eye Study. Ophthalmology. 1992; 99(1): 58-62, doi: 10.1016/s0161-6420(92)32011-1.

10. Zarbin MA. Current concepts in the pathogenesis of age-related macular degeneration. Arch Ophthalmol. 2004; 122(4): 598-614, doi: 10.1001/archopht.122.4.598, indexed in Pubmed: 15078679.

11. Zając-Pytrus HM, Pilecka A, Turno-Kręcicka A, et al. The dry form of age-related macular degeneration (AMD): The current concepts of pathogenesis and prospects for treatment. Adv Clin Exp Med. 2015; 24(6): 1099-1104, indexed in Pubmed: 26771984.

12. Kovalevskaya MA, Milyutkina SO. Efficacy of conservative treatment in patients with dry form of age-related macular degeneration. Vestn Oftalmol. 2015; 131(2): 81-88, indexed in Pubmed: 26080588.

13. Waheed NK, Moult EM, Fujimoto JG, et al. Optical coherence tomography angiography of dry age-related macular degeneration. Dev Ophthalmol. 2016; 56: 91-100, doi: 10.1159/000442784, indexed in Pubmed: 27023214.

14. Chong EWT, Kreis AJ, Wong TY, et al. Dietary omega-3 fatty acid and fish intake in the primary prevention of age-related macular degeneration: a systematic review and meta-analysis. Arch Ophthalmol. 2008; 126(6): 826-833, doi: 10.1001/archopht.126.6.826, indexed in Pubmed: 18541848. 
15. Bearelly $S$, Cousins $S W$. Fundus autofluorescence imaging in age-related macular degeneration and geographic atrophy. Adv Exp Med Biol. 2010; 664: 395-402, doi: 10.1007/978-1-4419-1399-9_45, indexed in Pubmed: 20238040

16. Batıoğlu F, Demirel $S$, Özmert E. Fundus autofluorescence imaging in age-related macular degeneration. Semin Ophthalmol. 2015; 30(1): 65-73, doi: 10.3109/08820538.2013.810285, indexed in Pubmed: 23952079.

17. Ly A, Nivison-Smith L, Assaad N, et al. Fundus autofluorescence in age-related macular degeneration. Optom Vis Sci. 2017; 94(2): 246-259, doi: 10.1097/0PX.0000000000000997, indexed in Pubmed: 27668639 .

18. http://www.roche.com/media/store/releases/med-cor-2017-09-08b. $\mathrm{htm}$.

19. Scharioth GB. New add-on intraocular lens for patients with age-related macular degeneration. J Cataract Refract Surg. 2015; 41(8): 1559-1563, doi: 10.1016/j.jcrs.2015.07.018, indexed in Pubmed: 26432110.

20. Nekolova J, Rozsival P, Sin M, et al. Scharioth Macula Lens: A new intraocular implant for low-vision patients with stabilized maculopathyfirst experience. Biomed Pap Med Fac Univ Palacky Olomouc Czech Repub. 2017; 161(2): 206-209, doi: 10.5507/bp.2017.014, indexed in Pubmed: 28452378.

21. Colby KA, Chang DF, Stulting RD, et al. Surgical placement of an optical prosthetic device for end-stage macular degeneration: the implantable miniature telescope. Arch Ophthalmol. 2007; 125(8): 1118-1121, doi: 10.1001/archopht.125.8.1118, indexed in Pubmed: 17698761.

22. Boyer D, Freund KB, Regillo C, et al. Long-term (60-month) results for the implantable miniature telescope: efficacy and safety outcomes stratified by age in patients with end-stage age-related macular degeneration. Clin Ophthalmol. 2015; 9: 1099-1107, doi: 10.2147/ OPTH.S86208, indexed in Pubmed: 26124633.
23. Yamamoto M, Kohno T, Shiraki K. Comparison of fundus autofluorescence of age-related macular degeneration between a fundus camera and a confocal scanning laser ophthalmoscope. Osaka City Med J. 2009; 55(1): 19-27, indexed in Pubmed: 19725431.

24. Deli A, Moetteli L, Ambresin A, et al. Comparison of fundus autofluorescence images acquired by the confocal scanning laser ophthalmoscope ( $488 \mathrm{~nm}$ excitation) and the modified Topcon fundus camera ( $580 \mathrm{~nm}$ excitation). Int Ophthalmol. 2013; 33(6): 635-643, doi: 10.1007/ s10792-013-9749-z, indexed in Pubmed: 23468053.

25. Park SP, Siringo FS, Pensec N, et al. Comparison of fundus autofluorescence between fundus camera and confocal scanning laser ophthalmoscope-based systems. Ophthalmic Surg Lasers Imaging Retina. 2013; 44(6): 536-543, doi: 10.3928/23258160-20131105-04, indexed in Pubmed: 24221461.

26. Khanifar AA, Lederer DE, Ghodasra JH, et al. Comparison of color fundus photographs and fundus autofluorescence images in measuring geographic atrophy area. Retina. 2012; 32(9): 1884-1891, doi: 10.1097/IAE.0b013e3182509778, indexed in Pubmed: 22547167.

27. Sayegh RG, Simader C, Scheschy U, etal. A systematic comparison of spectral-domain optical coherence tomography and fundus autofluorescence in patients with geographic atrophy. Ophthalmology. 2011; 118(9): 1844-1851, doi: 10.1016/.ophtha.2011.01.043, indexed in Pubmed: 21496928.

28. Simader C, Sayegh RG, Montuoro A, et al. A longitudinal comparison of spectral-domain optical coherence tomography and fundus autofluorescence in geographic atrophy. Am J Ophthalmol. 2014; 158(3): 557-566. e1, doi: 10.1016/j.ajo.2014.05.026, indexed in Pubmed: 24879944.

29. Graham KW, Chakravarthy U, Hogg RE, et al. Identifying features of early and late age-related macular degeneration: A comparison of multicolor versus traditional color fundus photography. Retina. 2017 [Epub ahead of print], doi: 10.1097/IAE.0000000000001777, indexed in Pubmed: 28834946. 
